\title{
Models for the Spread of White Pine Blister Rust
}

\author{
M.-R. Leung, ${ }^{\mathrm{a}, *}$ M. Kot ${ }^{\mathrm{a}}$ \\ ${ }^{a}$ Department of Applied Mathematics, University of Washington, Seattle, WA 98195, USA
}

\begin{abstract}
White pine blister rust (WPBR, Cronartium ribicola) is a fungal pathogen and a threat to whitebark pines (Pinus albicaulis). It has a complex life cycle that requires two hosts, a white pine and an alternate host, typically a currant or gooseberry (Ribes spp.). WPBR is transmitted between hosts by means of two types of airborne spores whose average dispersal distances differ by several orders of magnitude. In this paper, we introduce a discrete-time model based on the life cycle of WPBR. We then extend this model to include a continuous spatial domain, disease-induced mortality in the pines, and a latency period. After each extension, we find the pathogen's asymptotic speed of invasion analytically using exponential transforms and the method of steepest descent. Our results show that invasion speeds are strongly reduced by the latency period in the pine host. In addition, these speeds are highly dependent on the carrying capacity and infectiousness of each host type. If these parameters are sufficiently small, high mortality in pines may stop the spread of WPBR completely.
\end{abstract}

Keywords: Cronartium ribicola, integrodifference model, speed of invasion, exponential transform, steepest descent

2010 MSC: 92D25, 92D30

\section{Introduction}

The whitebark pine (Pinus albicaulis) is a keystone species of subalpine and tree-line habitats (Tomback et al., 2001; Zambino, 2010). In the coldest and driest sites, the whitebark pine is a climax species, but in protected areas, it is a seral species. Whitebark pines provide habitat for wildlife and protect watersheds by slowing snowmelt and stabilizing soil. Their large, indehiscent cones provide food for many species, including squirrels, bears, and Clark's Nutcrackers. These birds play a vital role in dispersing the cones' wingless seeds (Tomback et al., 2001).

Whitebark pines are found throughout western North America. The greatest threats to these trees are fire suppression, climate change, outbreaks of native

\footnotetext{
* Corresponding author

Email addresses: rleung@uw.edu (M.-R. Leung), mkot@uw.edu (M. Kot)
}

Preprint submitted to Elsevier

July 8, 2015 
mountain pine beetles, and the exotic pathogen white pine blister rust (WPBR, Cronartium ribicola) (Tomback and Achuff, 2010). WPBR was introduced from Europe in the early 20th century. This fungus was first discovered near Vancouver, BC in 1921 (Eastham, 1922; Güssow, 1922) and has since spread rapidly and decimated pine populations.

WPBR is capable of infecting all eight species of North American white pines (genus Pinus subgenus Strobus), and most species have been infested (Tomback and Achuff, 2010). The life cycle of WPBR is complex and requires an alternate or telial (teliospore-producing) host, in addition to a white pine serving as its primary or aecial (aeciospore-producing) host. The telial host is typically a member of the genus Ribes such as a currant or gooseberry, but some species of lousewort (Pedicularis spp.) and Indian paintbrush (Castilleja spp.) are also susceptible (McDonald et al., 2006). For brevity, we refer to all telial hosts as ribes.

WPBR continues to spread throughout the western United States, and understanding the factors affecting its rate of spread is important to protect remaining trees. In this paper, we develop a mathematical model for the dynamics of WPBR and its two hosts. We examine the behavior of this nonspatial model and calculate the asymptotic speed of disease invasion for increasingly complex spatial models that build on our nonspatial model. Examining how this invasion speed varies as we add and modify parameters gives insight into which factors are most influential.

The paper is organized as follows. In Section 2, we introduce the life cycle of WPBR. In sections 3-6, we develop a series of models of increasing complexity based on this life cycle. We analyze each model. For each spatial model, we calculate the speed of disease spread. Section 7 contains a discussion and some concluding remarks.

\section{Life cycle}

WPBR is a heteroecious fungus with a complex life cycle involving five distinct spore types. This life cycle is illustrated in Figure 1. In the spring, ribes are infected by long-range aeciospores released by infectious pines. After a short latency period of 2-3 weeks (Mielke, 1943; Spaulding, 1922), the ribes become infectious and release urediniospores (also known as uredospores) throughout the summer. These spores infect only ribes over relatively short distances. Towards the end of the year, infected ribes instead produce teliospores, which germinate to produce basidiospores. These are relatively short-ranged spores that infect pines by entering their needles. Infected pines develop cankers surrounded by pycniospores, or spermatia, which function as gametes to produce aeciospores the following spring (Maloy, 1997; Geils et al., 2010). The first aeciospores are typically released three to five years after infection and are produced annually thereafter.

Infection in pines begins in the needles and advances about 2 inches per year towards the trunk (Schwandt et al., 2013), leaving cankers and dead tissue behind. Flagging is symptomatic of branch girdling; cankers that reach and 
girdle the trunk cause topkill or death. There is no natural recovery, although cankers may be attacked by rodents, insects, or other fungi (Mielke, 1943), and minor infections may be stopped by pruning affected branches.

In ribes, WPBR infection is confined to foliage. Since most of these hosts are deciduous, infection is lost over winter when hosts shed their leaves. Thus, WPBR is annual in the telial host and perennial only in the aecial host (Pennington, 1925). Ribes species vary in their susceptibility and in their ability to transmit blister rust to other hosts (Mielke, 1943). Neither WPBR-induced mortality nor complete immunity have been observed in ribes, although severely infected leaves may fall early or die (Mielke, 1943).

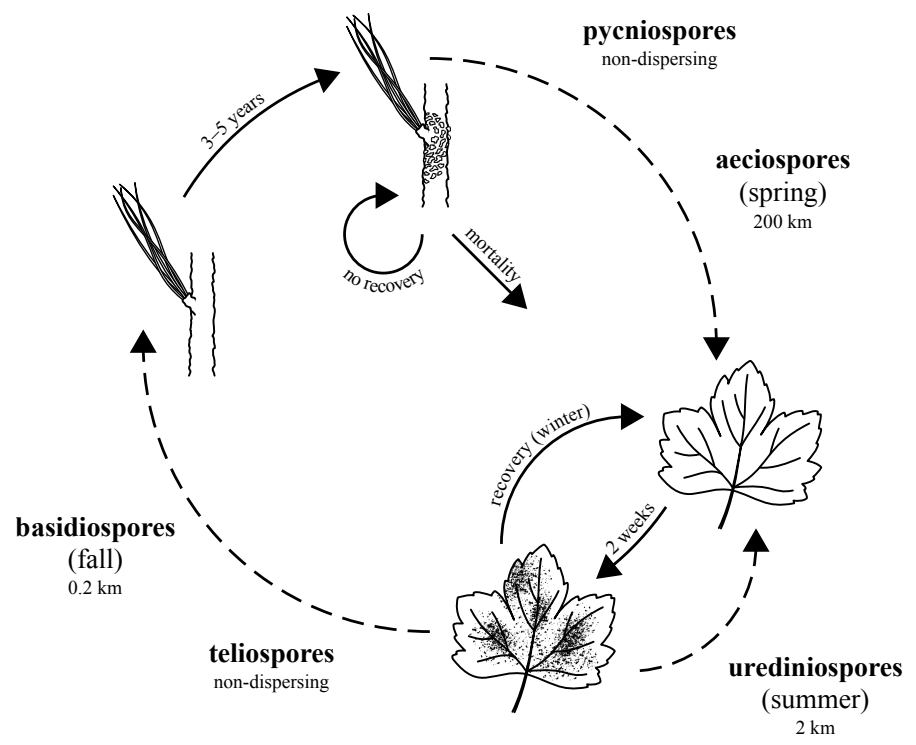

Figure 1: Spore-mediated transmission between hosts (dashed lines) and within-host dynamics (solid lines) of WPBR. In the spring, aeciospores transmit WPBR from infected pines to ribes. These spores are produced in tissue that contained pycniospores during the previous year. Infected ribes initially produce urediniospores that may infect other ribes. In late summer, they instead produce teliospores, which mature into basidiospores capable of infecting pines. Once infected, pines produce aeciospores annually after a 3-5 year latency period.

Our models are based on the WPBR life cycle, with some simplifications. We do not explicitly model the non-dispersing teliospores or pycniospores and we assume all ribes infections are caused by aeciospores rather than by urediniospores from other ribes. Aeciospores and basidiospores are assumed to be released at a single time in the spring or fall respectively. We also neglect varying susceptibilities and infectivities of different Ribes species and the effects of WPBR on ribes, such as leaf loss, that reduce basidiospore production. Since we are interested in a relatively short timescale, reproduction and natural mortality are not included.

Four models are described below. Since the WPBR life cycle is highly seasonal, we model time discretely, in half-years. We begin with a nonspatial model 
describing dynamics in a closed region. The next model is spatial and includes the dispersal kernels of WPBR spores on a continuous, linear domain. We then add disease-induced mortality in pines. Our last model includes both mortality and a latency period in newly infected pines before they become infectious.

The three spatial models are systems of integrodifference equations (IDEs). Powell and Zimmermann have used IDEs to estimate migration rates of whitebark pines (Powell and Zimmermann, 2004). See Allen and Ernest (2002) and Ríos-Soto et al. (2006) for other examples of disease models that use IDEs.

\section{Nonspatial model}

Our initial model is nonspatial and represents the Ribes-Pinus system in a closed patch. We initialize the system with ribes and pine populations at carrying capacity ( $K_{R}$ and $K_{P}$ respectively). We ignore reproduction and mortality so that the total population sizes remain constant, although the numbers of susceptible and infectious hosts may vary. We assume that there is no latency period in either host. Infected ribes recover annually, whereas the number of infected pines is nondecreasing since this model does not include recovery or mortality.

We assume that transmission of WPBR is governed by a saturating incidence function, so that as the number of infectious hosts increases, the number of new infections caused (in the opposite host type) increases, with diminishing returns, until it reaches a maximum of the opposite host's carrying capacity. The infectiousness of each species is governed by its half-saturation constant of infection $\left(h_{P}\right.$ or $\left.h_{R}\right)$. These parameters are positive and represent the number of infectious hosts required to infect half of the other host type. Therefore, larger values of these parameters indicate lower infectivity.

Our initial model is thus

$$
\begin{aligned}
R_{I, t+1 / 2} & =K_{R} \frac{P_{I, t}}{h_{P}+P_{I, t}}, \\
R_{S, t+1 / 2} & =K_{R}\left(1-\frac{P_{I, t}}{h_{P}+P_{I, t}}\right), \\
P_{I, t+1} & =P_{I, t}+P_{S, t} \frac{R_{I, t+1 / 2}}{h_{R}+R_{I, t+1 / 2}}, \\
P_{S, t+1} & =P_{S, t}\left(1-\frac{R_{I, t+1 / 2}}{h_{R}+R_{I, t+1 / 2}}\right) .
\end{aligned}
$$

Here, $P_{S, t}$ and $P_{I, t}$ are the number of susceptible and infected pines in the spring of year $t$, after new infections have occurred during the previous fall. The variables $R_{S, t+1 / 2}$ and $R_{I, t+1 / 2}$ are the number of susceptible and infected ribes in the fall of year $t$, after new infections in the preceding spring and summer, but before their winter recovery.

According to this model, infected pines infect a fraction of the susceptible population of ribes each year. Since ribes recover during the winter, this sus-

ceptible population is the carrying capacity, $K_{R}$. In the fall, these infected ribes 
infect a fraction of the susceptible pines, which are added to the previouslyinfected pines from earlier years. It is easy to see that the total populations remain constant, since $R_{S, t+1 / 2}+R_{I, t+1 / 2}=K_{R}$ and $P_{S, t+1}+P_{I, t+1}=P_{S, t}+P_{I, t}$, which was initialized at $K_{P}$.

To analyze model (1), we first write it in a seasonal form, with the size of each infected population depending solely on the size of the other infected population in the previous season. Solving infected ribes equation (1a) for $P_{I, t}$ gives

$$
P_{I, t}=\frac{h_{P} R_{I, t+1 / 2}}{K_{R}-R_{I, t+1 / 2}} .
$$

Using this and the fact that the susceptible pine population is determined by the infected and total populations $\left(P_{S, t}=K_{P}-P_{I, t}\right)$, we can rewrite infected pine equation (1c) to give $P_{I, t+1}$ as a function of $R_{I, t+1 / 2}$. In combination with infected ribes equation (1a), this gives the seasonal model

$$
\begin{aligned}
R_{I, t+1 / 2} & =\frac{K_{R} P_{I, t}}{h_{P}+P_{I, t}} \\
P_{I, t+1} & =\frac{K_{P} R_{I, t+1 / 2}}{h_{R}+R_{I, t+1 / 2}}+\frac{h_{R} h_{P} R_{I, t+1 / 2}}{\left(h_{R}+R_{I, t+1 / 2}\right)\left(K_{R}-R_{I, t+1 / 2}\right)} .
\end{aligned}
$$

These equations represent curves in the $R_{I}-P_{I}$ plane. They are plotted in Figure 2 along with a sample trajectory obtained by double-cobwebbing between them.

Double-cobwebbing, as described in Kot and Schaffer (1984), is a graphical method of solving a coupled system of seasonal equations. Reflecting a trajectory between the two curves gives populations at each time step. Intersections of the curves represent equilibria. In this model, a population at equilibrium is stable from year to year, but may still fluctuate seasonally because of the overwinter recovery of ribes.

We can examine the equilibria of seasonal model (3) most easily by reducing the system to a single equation. Substituting ribes equation (3a) into pine equation (3b) gives us a single equation for the infected pine population in year $t+1$ as a function of the pine population in year $t$,

$$
P_{I, t+1}=P_{I, t} \frac{h_{R} h_{P}+K_{R} K_{P}+h_{R} P_{I, t}}{h_{R} h_{P}+\left(K_{R}+h_{R}\right) P_{I, t}} .
$$

This model has two equilibria: a disease-free equilibrium $\left(P_{I, t}=0\right)$ and an infected equilibrium $\left(P_{I, t}=K_{P}\right)$. The stability of each equation is given by the slope of the right-hand side of annual model (4), evaluated at the equilibrium. For the disease-free equilibrium, the slope is

$$
1+\frac{K_{R} K_{P}}{h_{R} h_{P}},
$$




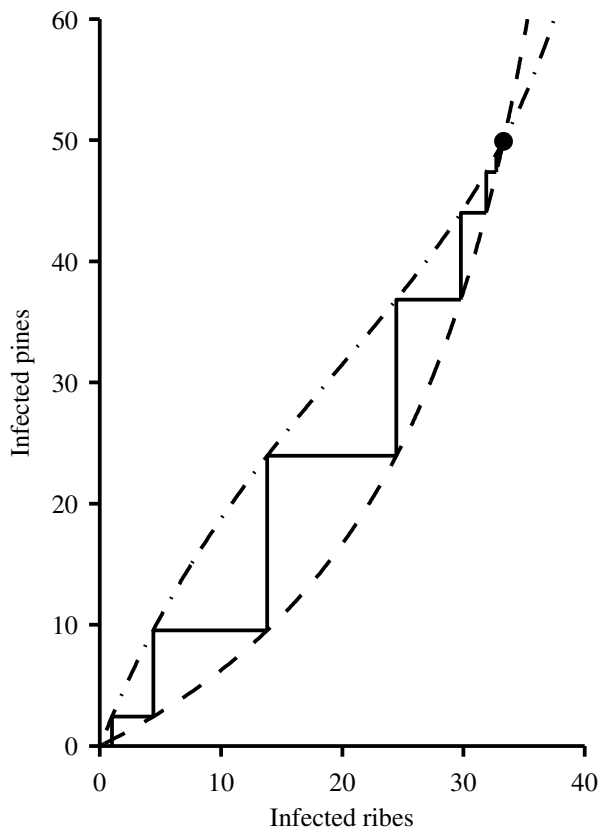

Figure 2: Double cobweb plot of the seasonal model (3), with the ribes curve (3a) as a dashed line and the pine curve $(3 \mathrm{~b})$ as a dashed-dotted line. The population trajectory (solid line) begins with a single infected ribes and no infected pines. The parameters are $h_{P}=h_{R}=25$ and $K_{R}=K_{P}=50$. 
which is greater than one. This equilibrium is unstable. At the infected equilibrium, the slope is

$$
\frac{h_{R}\left(h_{P}+K_{P}\right)}{h_{R}\left(h_{P}+K_{P}\right)+K_{R} K_{P}},
$$

which lies between zero and one. The infected equilibrium is asymptotically stable. Thus, this model predicts that once disease is introduced, it will eventually infect the entire pine population.

\section{Simple spatial model}

We now consider the model (1) on a continuous, one-dimensional, spatial domain. Our dependent variables and parameters are now densities rather than numbers of individuals. We allow host densities to vary as a function of their spatial position. We assume that infection is transmitted by airborne spores that disperse from infectious hosts according to a spore dispersal kernel. These kernels are probability density functions. We represent the aeciospore and basidiospore kernels by the functions $k_{A}(x-y)$ and $k_{B}(x-y)$ respectively. We assume that these kernels are symmetric functions of the displacement $x-y$. This assumption may not always hold in the study system, as spores are airborne and their dispersal patterns may be skewed due to prevailing winds and landscape features (Van Arsdel, 1965; Frank et al., 2008).

We also assume that an infected host contributes to incidence across space and that the distribution of this infection potential is identical to the host's fungal-spore dispersal kernel. The total infection potential at location $x$ is then given by integrating all of the contributions to $x$ from infectious hosts at points $y$. Mathematically, this integral is a convolution of the spore kernel with the density of infectious hosts. The numbers of new infections in the spatial model depend on the infection potentials given by these convolution integrals, instead of depending directly on the infectious populations, as in nonspatial model (1).

This method of modeling disease transmission with a convolution integral and without explicitly including spores or other infectious agents is standard in epidemic models with stationary hosts. Doing so allows for easy comparison between nonspatial and spatial versions of the same model. For more details, please see Mollison (1972) and Medlock and Kot (2003).

The resulting model is a system of four integrodifference equations. Since the total population densities are fixed, however, the densities of susceptible hosts are given by the difference between their carrying capacities and the densities of infectious hosts. Thus, we need only the two equations for infectious hosts at a location $x$,

$$
\begin{aligned}
R_{I, t+1 / 2}(x) & =\frac{K_{R} \int_{-\infty}^{\infty} k_{A}(x-y) P_{I, t}(y) d y}{h_{P}+\int_{-\infty}^{\infty} k_{A}(x-y) P_{I, t}(y) d y} \\
P_{I, t+1}(x) & =\frac{K_{P} \int_{-\infty}^{\infty} k_{B}(x-y) R_{I, t+1 / 2}(y) d y+h_{R} P_{I, t}(x)}{h_{R}+\int_{-\infty}^{\infty} k_{B}(x-y) R_{I, t+1 / 2}(y) d y}
\end{aligned}
$$




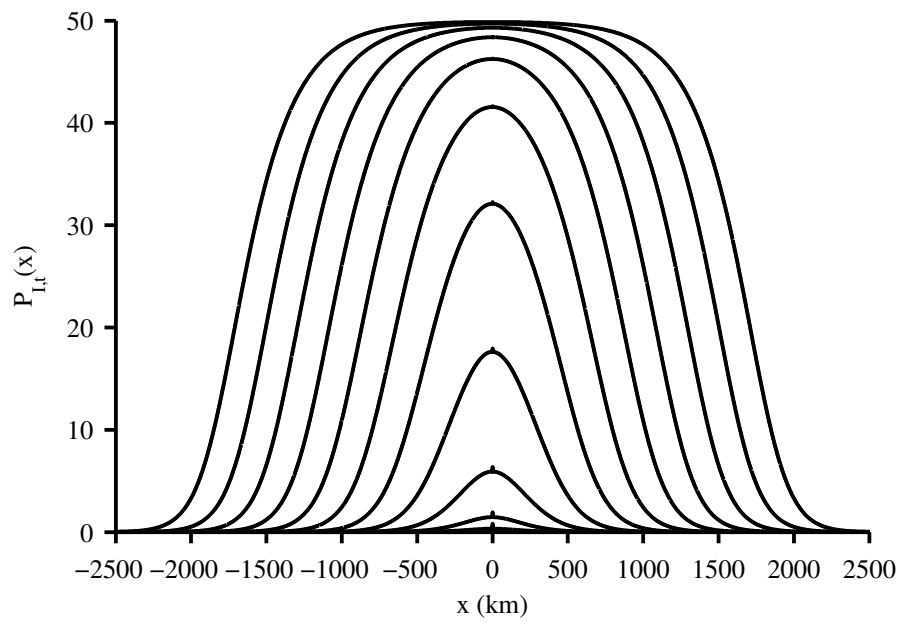

Figure 3: Annual infected pine populations, as predicted by simple spatial model (7), demonstrating the formation of a wave of infection. Populations are initially susceptible except for a low density $(0.5 / \mathrm{km})$ of infected ribes at the origin. We iterate the IDE for 20 years using the extended trapezoidal rule with $2^{18}+1$ nodes. The parameters are $h_{P}=h_{R}=25$, $K_{R}=K_{P}=50, a=0.015 \mathrm{~km}^{-1}$, and $b=15 \mathrm{~km}^{-1}$.

Here, we have taken the domain of integration to be the entire real line, $(-\infty, \infty)$.

Although this model is valid for any symmetric kernels $k_{A}$ and $k_{B}$, we will focus our attention on the Laplace kernels of the form

$$
k_{A}(x)=\frac{a}{2} \exp (-a|x|), \quad k_{B}(x)=\frac{b}{2} \exp (-b|x|) .
$$

Laplace kernels have been shown to adequately represent airborne spore dispersal data (Fitt et al., 1987; Okubo and Levin, 1989).

Larger values of the parameters $a$ and $b$ result in shorter average dispersal distances. To simulate our models, we take $a=0.015 \mathrm{~km}^{-1}$ and $b=15 \mathrm{~km}^{-1}$. For these parameters, $95 \%$ of aeciospores land within $200 \mathrm{~km}$ and $95 \%$ of basidiospores land within $0.2 \mathrm{~km}$. This is consistent with estimates for WPBR spores. Aeciospores usually land within several hundred kilometers (Mielke, 1943; Lundquist et al., 1992; Draper and Walla, 1993), while basidiospores typically land within several hundred meters (Mielke, 1943; Posey and Ford, 1924; Kimmey and Wagener, 1961; Tomback et al., 2001; Scheffer, 1997).

The dynamics of simple spatial model (7) are simulated in Figure 3. We initialize the model with a point release of a low density of infectious ribes into an otherwise susceptible population and plot the infected pine densities each year for 20 years. The disease soon infects all pines near the point release and forms a wave that spreads outwards in both directions from this central source.

\subsection{Analysis}

Suppose we introduce blister rust at a single point. If both spore kernels have exponentially bounded tails, the disease will form a traveling wave of infection 
that spreads at a constant rate throughout the spatial domain (Kot, 1992; Kot et al., 1996). Our goal is to determine this asymptotic speed.

We begin by linearizing spatial model (7) at the front of the wave, where the system is at its disease-free equilibrium and both infected populations are zero. We let $u_{t}$ and $v_{t}$ represent the distance of $R_{I, t}$ and $P_{I, t}$ from their equilibrium values. For our spatial model (7), this simply amounts to relabeling the dependent variables since they are zero at the disease-free equilibrium. The linearized model is then

$$
\begin{aligned}
u_{t+1 / 2}(x) & =\frac{K_{R}}{h_{P}} \int_{-\infty}^{\infty} k_{A}(x-y) v_{t}(y) d y, \\
v_{t+1}(x) & =\frac{K_{P}}{h_{R}} \int_{-\infty}^{\infty} k_{B}(x-y) u_{t+1 / 2}(y) d y+v_{t}(x) .
\end{aligned}
$$

To solve this system, we apply the exponential transformation, defined by Giffin (1975) as

$$
\hat{f}(s)=\int_{-\infty}^{\infty} f(x) e^{s x} d x .
$$

If the function $f(x)$ has exponentially bounded tails, this integral converges in some vertical strip in the complex $s$-plane.

This transformation possesses a convolution theorem that states that convolutions in the original spatial domain become products in the transformed domain. This makes the transformed system much simpler to solve.

Taking the exponential transform of linearized system (9) gives

$$
\begin{aligned}
\hat{u}_{t+1 / 2}(s) & =\frac{K_{R}}{h_{P}} M_{A}(s) \hat{v}_{t}(s), \\
\hat{v}_{t+1}(s) & =\frac{K_{P}}{h_{R}} M_{B}(s) \hat{u}_{t+1 / 2}(s)+\hat{v}_{t}(s),
\end{aligned}
$$

where

$$
M_{A}(s)=\hat{k}_{A}(s), \quad M_{B}(s)=\hat{k}_{B}(s),
$$

are the exponential transforms of the kernels. Since the kernels are probability density functions, their transforms are moment generating functions.

We can now manipulate this transformed system to obtain a difference equation for the transform of the infected pine density, $\hat{v}_{t}(s)$. Substituting the transformed infected ribes density (11a) into the transformed infected pine density (11b) gives

$$
\hat{v}_{t+1}(s)=\left(1+\frac{K_{R} K_{P}}{h_{R} h_{P}} M_{A}(s) M_{B}(s)\right) \hat{v}_{t}(s) .
$$

Group the parameters and moment generating functions as

$$
r=\frac{K_{R} K_{P}}{h_{R} h_{P}}, \quad M(s)=M_{A}(s) M_{B}(s) .
$$


The function $M(s)$ is the moment generating function for the convolution of the kernels $k_{A}(x)$ and $k_{B}(x)$. The parameter $r$ is a dimensionless quantity representing the ratio of the products of the carrying capacities to the products of the half-saturation constants of infection. Higher values of $r$ indicate the disease is better able to infect the pine population.

We now wish to find the function $\lambda(r, s)$ that gives a solution of difference equation (13) of the form

$$
\hat{v}_{t}(s)=\lambda^{t}(r, s) \hat{v}_{0},
$$

for some initial density $v_{0}$. For our simple spatial model, we set $\lambda(r, s)=$ $1+r M(s)$. This gives an exact solution of difference equation (13), but in general, expression (15) may represent an approximate solution valid for large $t$.

The solution in the original spatial domain is now given by the inverse transform,

$$
v_{t}(x)=\frac{v_{0}}{2 \pi i} \int_{-i \infty}^{+i \infty} \lambda^{t}(r, s) e^{-s x} d s
$$

We can use the method of steepest descent to approximate this integral for large $t$. With this method, we deform the contour of integration so that it passes through a critical point $s_{0}$, at which the real part of the integral reaches its maximum, and around which the real part declines rapidly. The general procedure is described by Kot and Neubert (2008). This method, however, requires the integrand to be written as a single exponent,

$$
v_{t}(x)=\frac{v_{0}}{2 \pi i} \int_{-i \infty}^{+i \infty} e^{t\left[K(r, s)-s \frac{x}{t}\right]} d s .
$$

In order to do this, we introduce the function $K(r, s)=\ln \lambda(r, s)$ that depends on the moment generating function $M(s)$, rather than using the cumulant generating function $\ln M(s)$ as Kot and Neubert do.

Following the method of steepest descent, we let $h(s)=K(r, s)-s \frac{x}{t}$ and look for a point $s_{0}$ such that the real part of $h$ has a nondegenerate saddle point at $s=s_{0}$. It is straightforward to show that this condition is satisfied if $h$ is analytic in a region of the plane containing the contour, with $h^{\prime}(s)=0$ and $h^{\prime \prime}(s) \neq 0$. The saddle point exists regardless of the sign of $K_{s s}$, but in our case $K$ is convex and $K_{s s}$ is positive. The convexity of $K$ follows from the fact that moment generating functions are log-convex (i.e., their logarithms are convex) and from the preservation of logarithmic convexity for sums of logconvex functions and for log-convex functions that are multiplied by positive real numbers.

We can then show that the path of integration should be parallel to the imaginary axis. The resulting approximation is

$$
v_{t} \approx \frac{v_{0} \lambda^{t}\left(r, s_{0}\right) e^{-s_{0} x}}{\sqrt{2 \pi t K_{s s}\left(r, s_{0}\right)}}
$$


for large $t$, where $K_{s s}$ is the second-order partial derivative of $K$ with respect to $s$, and $s_{0}$ is defined by the equality

$$
\frac{\lambda_{s}\left(r, s_{0}\right)}{\lambda\left(r, s_{0}\right)}=\frac{x}{t}
$$

where $\lambda_{s}$ is the partial derivative of $\lambda$ with respect to $s$. Thus, $s_{0}$ is a critical point of the exponent in the inverse transform (17).

Using this approximation, we can calculate the asymptotic speed of disease invasion. Suppose the infection is only detected above a threshold infected population density $v_{c}$, which occurs at some position $x=x\left(t, v_{c}\right)>0$ at time $t$. We then set $v_{t}=v_{c}$ and solve the approximate infected pine density (18) for the ratio $x / t$ to get

$$
\frac{x}{t}=\frac{1}{s_{0}}\left[K\left(r, s_{0}\right)-\frac{1}{t} \ln \frac{v_{c}}{v_{0}} \sqrt{2 \pi t K_{s s}\left(r, s_{0}\right)}\right] .
$$

If this ratio converges as $t$ increases, then so do $s_{0}$ and $K_{s s}\left(r, s_{0}\right)$. The limit of the ratio is the asymptotic speed $c$,

$$
c=\lim _{t \rightarrow \infty} \frac{x}{t}=\frac{K\left(r, s_{0}\right)}{s_{0}}=\frac{\ln \lambda\left(r, s_{0}\right)}{s_{0}} .
$$

For model (7), we set $\lambda(r, s)=1+r M(s)$, so that formula (21) gives the speed $c$ as a function of both the (given) parameter $r$ and the critical point $s_{0}$. Since both $c$ and $s_{0}$ are unknown, we need a second equation.

If $c$ exists, then by equation (19), it equals the ratio of $\lambda_{s}$ to $\lambda$ at the critical point $s_{0}$. Using this fact and rewriting equation (21) for the speed to solve for $\lambda\left(r, s_{0}\right)$, we have a parametric system for $c$,

$$
c=\frac{\lambda_{s}\left(r, s_{0}\right)}{\lambda\left(r, s_{0}\right)}, \quad e^{s_{0} c}=\lambda\left(r, s_{0}\right) .
$$

Note that the first equation can also be obtained by multiplying the speed equation (21) by $s_{0}$ and differentiating with respect to $s_{0}$. This implies that $s_{0}$ is a double root.

We now rewrite this system using the definition of $\lambda(r, s)$. Substituting the first equation of system (22) into the second gives the system

$$
\begin{aligned}
& c=\frac{r M^{\prime}\left(s_{0}\right)}{1+r M\left(s_{0}\right)}, \\
& r=\frac{1}{M\left(s_{0}\right)}\left[\exp \left(\frac{s_{0} r M^{\prime}\left(s_{0}\right)}{1+r M\left(s_{0}\right)}\right)-1\right] .
\end{aligned}
$$

We wish to use these equations to find the asymptotic rate of disease spread, $c$, as a function of $r$. To do so, we use a root finder, such as Brent's method, to solve equation (23b), either for $r$ in terms of $s_{0}$ or for $s_{0}$ in terms of $r$. We then use $r, s_{0}$, and equation (23a) to determine $c$. 


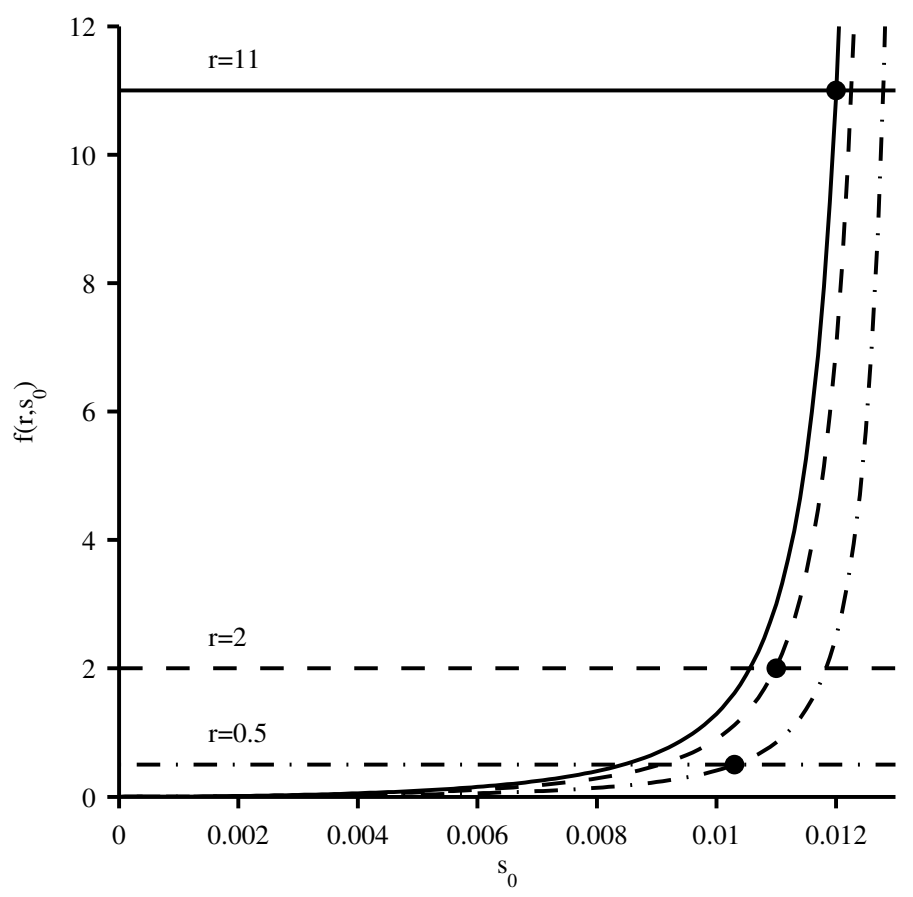

Figure 4: Plot of (23b), written as $r=f\left(r, s_{0}\right)$ and shown as a function of $s_{0}$. For each $r$-value, there is both a horizontal line at $r$ and a curve corresponding to $f\left(r, s_{0}\right)$, plotted with the same line style. Dots indicate intersection points corresponding to positive solutions of (23b). As $r$ decreases, the curves become increasingly flat near the origin, leading to a positive minimum value of $s_{0}$ at the intersection points. As $r$ increases, the intersection points approach $\min (a, b)$. Here we assume Laplace kernels with parameters $a=0.015 \mathrm{~km}^{-1}$ and $b=15 \mathrm{~km}^{-1}$.

Alternatively, we can solve (23b) graphically by plotting both sides of the equation and looking for intersections between them. We can plot these curves either as a function of $s_{0}$, shown in Figure 4, or as a function of $r$, shown in Figure 5. These plots illustrate several important properties of solutions of this equation.

First, there is a maximum possible $s_{0}$, since if this value exceeds either of the Laplace parameters $a$ or $b$, the exponential transform (10) does not converge. As shown in Figure 4, as $r$ increases, the corresponding value of $s_{0}$ approaches this limit. Second, although $r=0$ is always a root of (23b), this value is not biologically meaningful, since it implies that the carrying capacity of one or both populations is zero. Figure 5 shows that there is a threshold value of $s_{0}$, above which there is one positive root $r$ for each $s_{0}$. In both cases, each positive value of $r$ corresponds to exactly one positive value of $s_{0}$. We prove this result more rigorously in Appendix 7.

We can solve the system (23) numerically for a range of $r$-values. The resulting relationship between $r$ and $c$ is given in Figure 6. This plot shows how 


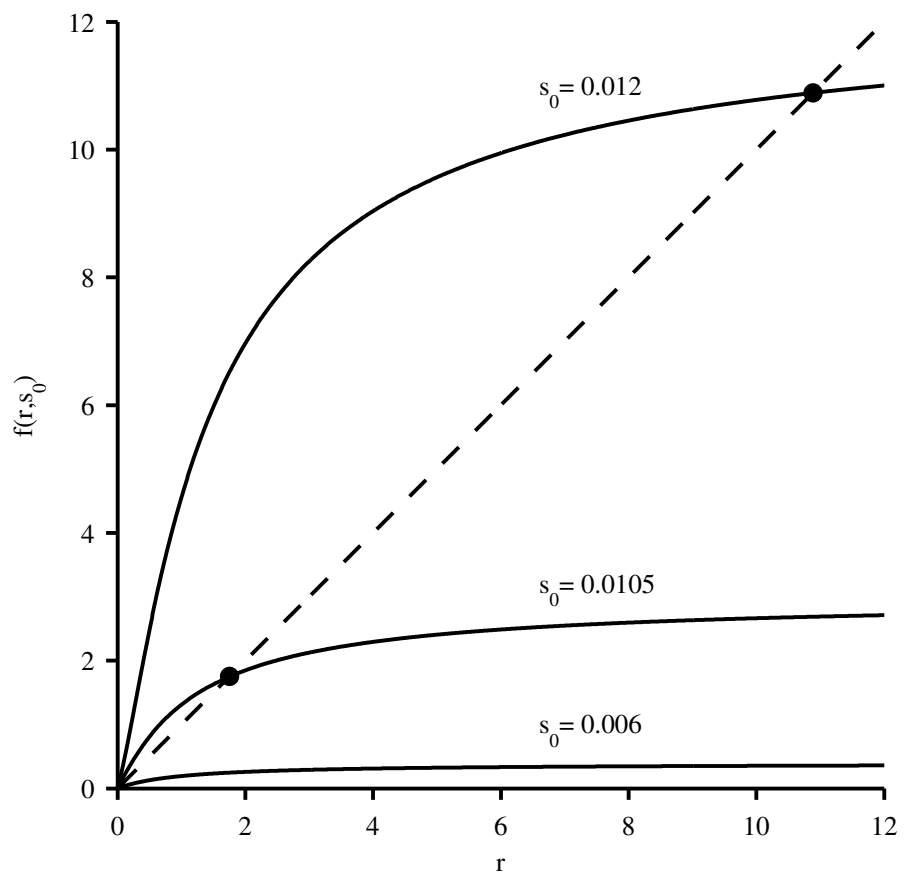

Figure 5: Plot of (23b), written as $r=f\left(r, s_{0}\right)$ and shown as a function of $r$. Dots indicate intersection points corresponding to positive solutions of (23b). For small $s_{0}$, the function $f$ is concave down with initial slope less than 1 , so $r=f\left(r, s_{0}\right)$ has no solutions. For larger $s_{0}$ (in this case, $\left.s_{0}>0.0087\right), f$ has initial slope greater than 1 , and has exactly one solution. For sufficiently large $s_{0}$, the curve is initially concave up. Here we assume Laplace kernels with parameters $a=0.015 \mathrm{~km}^{-1}$ and $b=15 \mathrm{~km}^{-1}$. 


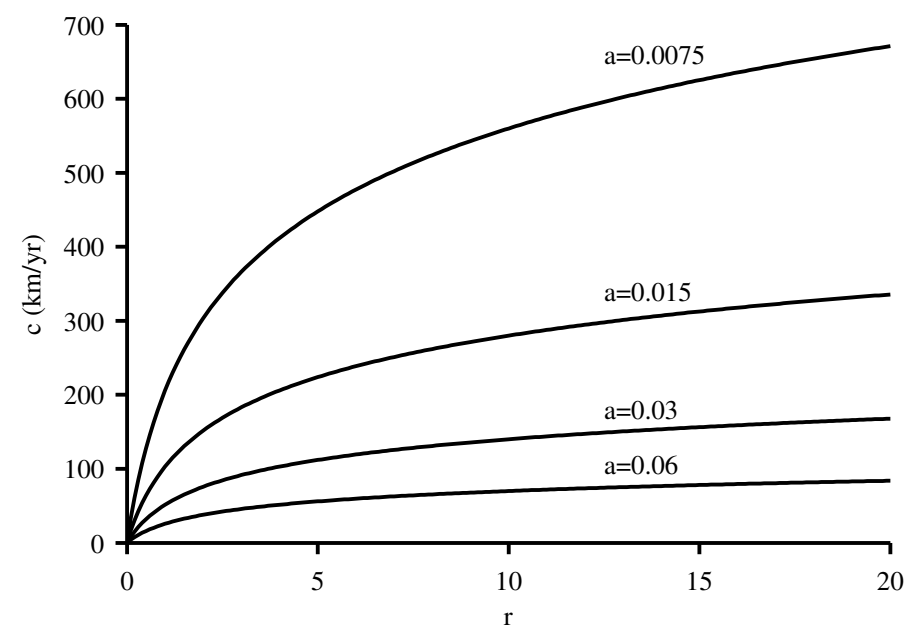

Figure 6: Plot of the speed of invasion $(c)$ for varying $r$-values, assuming Laplace kernels with parameters $b=15 \mathrm{~km}^{-1}$ and $a=0.0075,0.015,0.03$, or $0.06 \mathrm{~km}^{-1}$.

the speed of disease invasion changes in response to the populations' carrying capacities and half-saturation constants of infection. We again assume $k_{A}(x)$ and $k_{B}(x)$ are Laplace kernels, given in (8), which have moment generating functions

$$
M_{A}(s)=\frac{a^{2}}{a^{2}-s^{2}}, \quad M_{B}(s)=\frac{b^{2}}{b^{2}-s^{2}} .
$$

We fix the basidiospore dispersal parameter $b$ at $15 \mathrm{~km}^{-1}$, but different aeciospore dispersal parameters are shown as different curves. For larger values of $a$, aeciospores travel shorter distances on average and the speed of disease invasion is slower.

Convergence to the asymptotic speed is rapid. Taking $a=0.015 \mathrm{~km}^{-1}$, $b=15 \mathrm{~km}^{-1}$, and $r=4$, we use system (23) to find the asymptotic speed, $c \approx 206 \mathrm{~km} / \mathrm{yr}$. As shown in Figure 7, once the wave of infection begins to spread, it quickly reaches its asymptotic speed. The short-term invasion speed in this figure is highly dependent on the threshold density of infected pines we chose to define the outer edge of the invasion, as well as the initial condition of the invasion.

\section{Spatial model with mortality}

Although mature pines may survive for years after WPBR infection, younger trees often die quickly. To model this effect, we suppose a constant fraction $m \in[0,1]$ of infected pines die each year. As a result, the total pine density is no longer fixed at its carrying capacity, and we must model the susceptible density explicitly. Adding the equation for the density of the susceptible pines, 


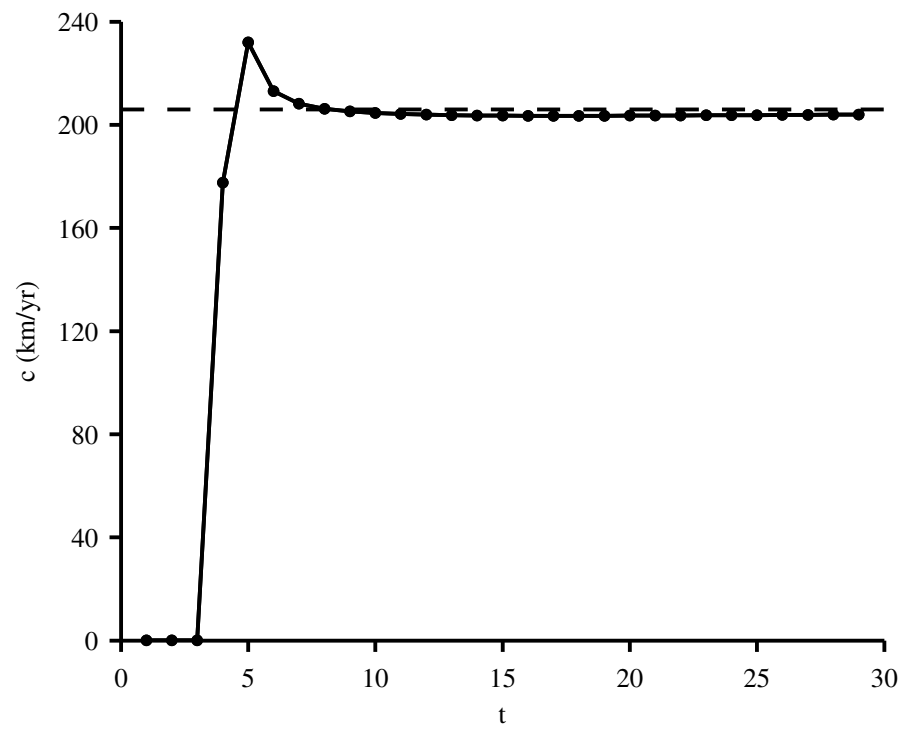

Figure 7: Annual speed of the invasion predicted by simple spatial model (7) compared to its asymptotic speed $c \approx 206 \mathrm{~km} / \mathrm{yr}$ (dotted line). Speeds are computed by tracking a threshold density of 0.05 infected pines $/ \mathrm{km}$. Invasions travel near their asymptotic speed almost immediately. Populations are initially susceptible except for a low density $(0.5 / \mathrm{km})$ of infected pines at the origin. We iterate the IDE using the extended trapezoidal rule with $2^{19}+1$ nodes. The parameters are $h_{P}=h_{R}=25, K_{R}=K_{P}=50, a=0.015 \mathrm{~km}^{-1}$, and $b=15 \mathrm{~km}^{-1}$. The overall speed is sensitive to the number of nodes and the short-term speed is sensitive to the infection threshold and initial condition. 
$P_{S, t}(x)$, back into spatial model (7) and adding fixed mortality gives the threedimensional system

$$
\begin{aligned}
R_{I, t+1 / 2}(x) & =\frac{K_{R} \int_{-\infty}^{\infty} k_{A}(x-y) P_{I, t}(y) d y}{h_{P}+\int_{-\infty}^{\infty} k_{A}(x-y) P_{I, t}(y) d y} \\
P_{I, t+1}(x) & =(1-m) P_{I, t}(x)+P_{S, t}(x) \frac{\int_{-\infty}^{\infty} k_{B}(x-y) R_{I, t+1 / 2}(y) d y}{h_{R}+\int_{-\infty}^{\infty} k_{B}(x-y) R_{I, t+1 / 2}(y) d y} \\
P_{S, t+1}(x) & =P_{S, t}(x)\left(1-\frac{\int_{-\infty}^{\infty} k_{B}(x-y) R_{I, t+1 / 2}(y) d y}{h_{R}+\int_{-\infty}^{\infty} k_{B}(x-y) R_{I, t+1 / 2}(y) d y}\right)
\end{aligned}
$$

Although this system contains an additional equation for the susceptible pines, calculation of the asymptotic rate of disease spread $(c)$ follows as described in Section 4.1. We first linearize around the disease-free equilibrium, where $P_{S, t}=K_{P}$ and both infected densities are zero. We then transform the system and substitute the equation for the transformed infected ribes density $\left(\hat{u}_{t}\right)$ into those for susceptible and infected pines $\left(\hat{v}_{t}\right.$ and $\left.\hat{w}_{t}\right)$. This gives a system of two equations,

$$
\hat{v}_{t+1}=\hat{v}_{t}-r M(s) \hat{w}_{t}, \quad \hat{w}_{t+1}=(1-m+r M(s)) \hat{w}_{t} .
$$

The second of these equations is a first-order difference equation for the transform of the infected pine density. By setting $\lambda(r, s)=1-m+r M(s)$, we can write its solution as $\hat{w}_{t}(s)=\lambda^{t}(r, s) \hat{w}_{0}$. Substituting this $\lambda(r, s)$ into parametric system (22) and solving for $r$ as before gives the parametric equations

$$
\begin{aligned}
c & =\frac{r M^{\prime}\left(s_{0}\right)}{1-m+r M\left(s_{0}\right)}, \\
r & =\frac{1}{M\left(s_{0}\right)}\left[\exp \left(\frac{s_{0} r M^{\prime}\left(s_{0}\right)}{1-m+r M\left(s_{0}\right)}\right)-(1-m)\right] .
\end{aligned}
$$

We can show that (27b) has a single positive solution for all $s_{0} \geq 0$ (see Appendix 7 ), so that the numerical method for solving system (27) is the same as before.

The relationship between $c$ and $r$ for varying $m$-values is plotted in Figure 8. If the disease does not kill its hosts, then $m=0$ and spatial model with mortality (25) simplifies to simple spatial model (7), described in Section 4. Positive mortality rates do slow the speed of invasion, although not considerably. However, if $r<m$, the disease kills its hosts faster than they are able to propagate it through the system and it is unable to spread.

In reality, the life expectancy of an infected tree is highly variable and depends on the size of the tree, severity of infection, and location of cankers. Field et al. (2012) divided the pine population into size classes and provides estimates of the disease-induced mortality rates for each class. We can use the percentage of the population in each class at the disease-free equilibrium to take a weighted average of these mortality rates. This gives an approximate annual mortality rate for the entire population of 0.27 . 


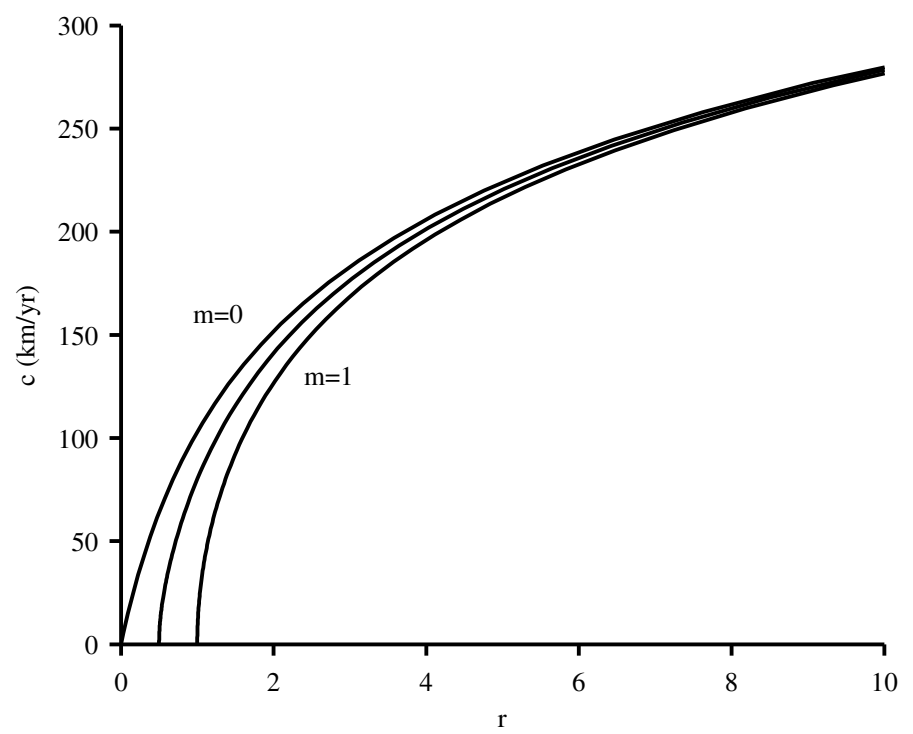

Figure 8: Plot of the speed of invasion $(c)$ versus $r$, assuming Laplace kernels with parameters $a=0.015 \mathrm{~km}^{-1}$ and $b=15 \mathrm{~km}^{-1}$. Mortality rates $m=0,0.5$, and 1 are shown. For $r<m$, the speed is zero and the disease is unable to spread.

It is interesting to note that if WPBR is always fatal to its aecial host after a single year $(m=1)$, parametric equations $(27)$ simplify to those for the onedimensional, single-species model analyzed by Kot et al. (1996). In other words, the infection and mortality dynamics in spatial model (25) are analogous to the growth and dissemination dynamics of a population with distinct sedentary and dispersal stages.

\section{Spatial model with mortality and exposed class}

Pines infected with WPBR do not immediately become infectious. Instead, they experience a latency period of several years before first producing aeciospores. We model this by adding an additional function, $P_{E, t}(x)$, representing the density of exposed pines. These pines do not suffer disease-induced mortality nor do they contribute to infection of ribes. A fraction $p$ of these pines remain exposed each year, while the remainder $(1-p)$ move into the infectious 
class. This gives the model

$$
\begin{aligned}
R_{I, t+1 / 2}(x) & =\frac{K_{R} \int_{-\infty}^{\infty} k_{A}(x-y) P_{I, t}(y) d y}{h_{P}+\int_{-\infty}^{\infty} k_{A}(x-y) P_{I, t}(y) d y}, \\
P_{S, t+1}(x) & =P_{S, t}(x)\left(1-\frac{\int_{-\infty}^{\infty} k_{B}(x-y) R_{I, t+1 / 2}(y) d y}{h_{R}+\int_{-\infty}^{\infty} k_{B}(x-y) R_{I, t+1 / 2}(y) d y}\right) \\
P_{E, t+1}(x) & =p P_{E, t}(x)+P_{S, t}(x) \frac{\int_{-\infty}^{\infty} k_{B}(x-y) R_{I, t+1 / 2}(y) d y}{h_{R}+\int_{-\infty}^{\infty} k_{B}(x-y) R_{I, t+1 / 2}(y) d y}, \\
P_{I, t+1}(x) & =(1-p) P_{E, t}(x)+(1-m) P_{I, t}(x) .
\end{aligned}
$$

Linearizing about the disease-free equilibrium, at which all variables are zero except $P_{S, t}=K_{P}$, and taking the exponential transform of this model gives a system of four difference equations. We can substitute the transform of the infected ribes density into those of the susceptible and exposed pines. This reduces the transformed system to three difference equations, corresponding to the transformed and linearized susceptible, exposed, and infected pine densities, $\left(\hat{u}_{t}, \hat{v}_{t}\right.$, and $\left.\hat{w}_{t}\right)$,

$\hat{u}_{t+1}=\hat{u}_{t}-r M(s) \hat{w}_{t}, \quad \hat{v}_{t+1}=p \hat{v}_{t}+r M(s) \hat{w}_{t}, \quad \hat{w}_{t+1}=(1-p) \hat{v}_{t}+(1-m) \hat{w}_{t}$.

The last two equations do not depend on the first equation and decouple. From these two equations we obtain the characteristic equation

$$
\lambda^{2}-(p+1-m) \lambda+(1-m) p-(1-p) r M(s)=0 .
$$

Although this is second-order and has two roots, we can approximate the solution of the two difference equations by setting $\lambda(r, s)$ equal to the larger root,

$$
\lambda(r, s)=\frac{p+1-m+\sqrt{(p-1+m)^{2}+4(1-p) r M(s)}}{2} .
$$

We can then write, for example, $\hat{v}_{t}(s) \approx \lambda^{t}(r, s) \hat{v}_{0}$. This approximation is valid for large $t$, since the effect of the smaller root will become negligible over time. The procedure for computing the inverse transform is exactly as before, and since the asymptotic speed of invasion also holds as $t$ increases, making this approximation does not invalidate our results.

Figure 9 gives solutions of parametric system (22) using this definition of $\lambda(r, s)$. Although adding a latency period will not alter pathogen persistence, as mortality can, increasing the length of the latency period can dramatically reduce the speed of invasion.

Based on his work with P. monticola, Lachmund (1933) found that $35 \%$ of infected trees begin producing aeciospores in the third spring after infection, $43 \%$ in the fourth spring, $18 \%$ in the fifth spring, and $3 \%$ in the sixth and seventh springs. Assuming similar values for $P$. albicaulis, the typical latency period is approximately 4 years, which is represented by the curve $p=0.75$ in Figure 9. 


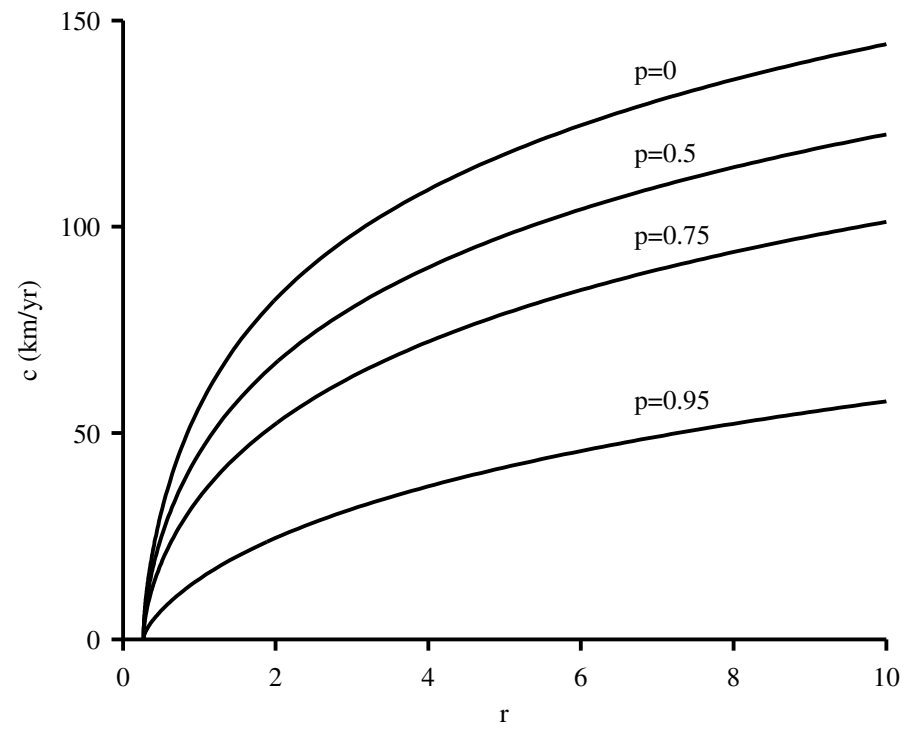

Figure 9: Plot of the speed of invasion $(c)$ versus $r$, assuming a mortality rate $m=0.27$ and Laplace kernels with parameters $a=0.015 \mathrm{~km}^{-1}$ and $b=15 \mathrm{~km}^{-1}$. Values for $p$ of $0,0.5$, 0.75 and 0.95 are shown. Larger values correspond to longer average latency periods.

\section{Discussion}

We have developed four models for WPBR, beginning with a nonspatial model and adding space, disease-induced mortality, and a latency period. The nonspatial model predicts that once WPBR has been introduced, it will eventually infect all pines in a given area, reaching an asymptotically stable endemic equilibrium. This is the case in the simple spatial model as well, although here the disease spreads throughout the system in the form of a traveling wave that converges to a constant speed.

Without mortality in pines, the disease always persists and spreads, but with disease-induced mortality, the invasion can be stopped if the mortality rate $m$ is greater than the parameter $r$. In this case, infected trees die faster than they are able to propagate the disease. However if $r$ is larger than 1, even mortality after one year of infection is insufficient to stop the disease. As $r$ increases, the effect of mortality becomes increasingly negligible.

Conversely, a latency period has a much greater slowing effect on invasion speeds, even for larger $r$, but this alone cannot stop the spread of disease.

In all cases, the speed was an increasing, concave-down function of the parameter $r$. If $r$ is small, even small reductions can have a large impact on the speed of invasion. This parameter depends on the carrying capacities and half-saturation constants of infection for both pines and ribes. It could be reduced by reducing the Ribes carrying capacity or by reducing the number of new infections caused by each infectious host.

We demonstrated a novel technique for calculating the speed of invasion 
in systems of IDEs. By taking the exponential transform, systems (7), (25), and (28) become much easier to solve. We can then approximate the inverse transform using the method of steepest descent, and use the result to calculate the asymptotic rate of disease spread. Despite the increasing complexity and number of equations in the models, this calculation was straightforward and demonstrates the power and versatility of this technique. It can be applied to many IDE models and greatly simplifies the computation of invasion or dispersal speeds.

Future work on these models should focus on adding more biological realism. In particular, since the rate at which disease progresses in trees is strongly correlated to their size, differentiating size classes in Pinus would provide greater insight into how the disease spreads. In addition, adding reproduction in pines is important for these models to be accurate on longer timescales and to explore how forests regenerate after a WPBR invasion. Finally, a more complex model could remove the assumption of spatial homogeneity and allow for skewed spore dispersal, as well as reflect the often-fragmented habitat of whitebark pines.

\section{Acknowledgements}

We are very grateful to our two anonymous reviewers for their insightful comments. This material is based upon work supported by the ARCS Foundation Seattle chapter and the National Science Foundation (NSF) Graduate Research Fellowship Program under Grant Nos. DGE-0718124 and DGE-1256082 to M.-R.L. and by NSF grant DMS-1308365 to M.K. Any opinions, findings, and conclusions or recommendations expressed in this material are those of the authors and do not necessarily reflect the views of the NSF.

\section{Appendix A. Finding $r$ given $s_{0}$}

Equation (27b) states that $r=f\left(r, s_{0}\right)$, where

$$
f\left(r, s_{0}\right)=\frac{1}{M\left(s_{0}\right)}\left[\exp \left(\frac{s_{0} r M^{\prime}\left(s_{0}\right)}{1-m+r M\left(s_{0}\right)}\right)-(1-m)\right] .
$$

Equation (23b), corresponding to our initial spatial model (7) without mortality, is a special case of this equation with $m=0$.

Let $s_{0} \geq 0$ be fixed. Since $M(s)$ is a moment generating function, $M\left(s_{0}\right) \geq 1$ and $M^{\prime}\left(s_{0}\right) \geq 0$.

If the mortality rate $m$ is 1 , the function $f\left(r, s_{0}\right)$ simplifies to

$$
f\left(r, s_{0}\right)=\frac{1}{M\left(s_{0}\right)}\left[\exp \left(\frac{s_{0} M^{\prime}\left(s_{0}\right)}{M\left(s_{0}\right)}\right)\right]=g\left(s_{0}\right),
$$

which is independent of $r$. Then the equation $r=g\left(s_{0}\right)$ has a positive solution $r$ for all $s_{0}$. 
Now suppose $m<1$. Since

$$
f\left(0, s_{0}\right)=m / M\left(s_{0}\right),
$$

$f\left(r, s_{0}\right)$ is initially nonnegative, and since

$$
\lim _{r \rightarrow \infty} f\left(r, s_{0}\right)=\frac{1}{M\left(s_{0}\right)}\left[\exp \left(\frac{s_{0} M^{\prime}\left(s_{0}\right)}{M\left(s_{0}\right)}\right)-(1-m)\right] \geq 0,
$$

this function converges to a nonnegative limit.

The slope of $f$ is

$$
f_{r}\left(r, s_{0}\right)=\frac{s_{0}(1-m) M^{\prime}\left(s_{0}\right)}{M\left(s_{0}\right)\left(1-m+r M\left(s_{0}\right)\right)^{2}}\left[\exp \left(\frac{s_{0} r M^{\prime}\left(s_{0}\right)}{1-m+r M\left(s_{0}\right)}\right)\right],
$$

which is nonnegative, and its concavity is given by

$$
\begin{aligned}
f_{r r}\left(r, s_{0}\right)= & {\left[s_{0}(1-m) M^{\prime}\left(s_{0}\right)-2(1-m) M\left(s_{0}\right)-2 r M^{2}\left(s_{0}\right)\right] } \\
& \times \frac{s_{0}(1-m) M^{\prime}\left(s_{0}\right) \exp \left(\frac{s_{0} r M^{\prime}\left(s_{0}\right)}{1-m+r M\left(s_{0}\right)}\right)}{M\left(s_{0}\right)\left(1-m+r M\left(s_{0}\right)\right)^{4}} .
\end{aligned}
$$

Thus, $f\left(r, s_{0}\right)$ is nondecreasing and, for all sufficiently large $r$, the last term in the square brackets of (A.6) ensures that it is concave down.

At the origin, $f\left(r, s_{0}\right)$ has slope

$$
f_{r}\left(0, s_{0}\right)=\frac{s_{0} M^{\prime}\left(s_{0}\right)}{M\left(s_{0}\right)(1-m)},
$$

which is nonnegative, and concavity

$$
f_{r r}\left(0, s_{0}\right)=\left[s_{0} M^{\prime}\left(s_{0}\right)-2 M\left(s_{0}\right)\right] \frac{s_{0} M^{\prime}\left(s_{0}\right)}{M\left(s_{0}\right)(1-m)^{2}},
$$

which could be either positive or negative.

Then, if $f\left(r, s_{0}\right)$ is initially concave up, it has one inflection point, since setting concavity equation (A.8) equal to zero implies

$$
r=\frac{1-m}{M\left(s_{0}\right)}\left(\frac{s_{0} M^{\prime}\left(s_{0}\right)}{2 M\left(s_{0}\right)}-1\right) .
$$

Suppose $f\left(r, s_{0}\right)$ has no positive inflection points. Then it is always concave down. If $m>0$ such that $f$ is initially positive, or if $m=0$ and $f$ has initial slope greater than 1 , then $r=f\left(r, s_{0}\right)$ has a single positive solution $r$. Otherwise, it has only the zero solution.

If $f\left(r, s_{0}\right)$ has a positive inflection point, then by (A.8) it is initially concave up, and by (A.7), its initial slope $f_{r}\left(0, s_{0}\right)$ satisfies the inequality

$$
f_{r}\left(0, s_{0}\right) \geq \frac{2}{1-m} \geq 2 .
$$

Thus, $r=f\left(r, s_{0}\right)$ has exactly one positive solution.

These results are summarized in Table A.1 and shown graphically in Figure 5. If $m>0$, there is a positive solution of $r=f\left(r, s_{0}\right)$. If $m=0$, then $f\left(r, s_{0}\right)$ has a positive solution iff $s_{0}$ is large enough such that $f_{r}\left(0, s_{0}\right)>1$. 


\begin{tabular}{ccc}
\hline Condition & $\begin{array}{c}\text { Inflection } \\
\text { points }\end{array}$ & $\begin{array}{c}\text { Positive } \\
\text { solutions }\end{array}$ \\
\hline$s_{0} M^{\prime}\left(s_{0}\right)<(1-m) M\left(s_{0}\right)$ & 0 & 0 \\
$(1-m) M\left(s_{0}\right)<s_{0} M^{\prime}\left(s_{0}\right)<2 M\left(s_{0}\right)$ & 0 & 1 \\
$2 M\left(s_{0}\right)<s_{0} M^{\prime}\left(s_{0}\right)$ & 1 & 1 \\
\hline
\end{tabular}

Table A.1: Features of $f\left(r, s_{0}\right)$ and positive solutions of $r=f\left(r, s_{0}\right)$, ordered by increasing $s_{0}$. Note that $r=0$ is always a solution but is not biologically relevant.

\section{References}

Allen, L.J.S., Ernest, R.K., 2002. The impact of long-range dispersal on the rate of spread in population and epidemic models, in: Castillo-Chavez, C., Blower, S., van den Driessche, P., Kirschner, D., Yakubu, A.A. (Eds.), Mathematical Approaches for Emerging and Reemerging Infectious Diseases: An Introduction. Springer, New York, NY. IMA Volumes in Mathematics and its Applications, pp. 183-197.

Draper, M.A., Walla, J.A., 1993. First report of Cronartium ribicola in North Dakota. Plant Disease 77, 952.

Eastham, J.W., 1922. Report of provincial plant pathologist, Vancouver, in: Province of British Columbia, Sixteenth Annual Report of the Department of Agriculture for the Year 1921. W.H. Cullin, Victoria, pp. 64-69.

Field, S.G., Schoettle, A.W., Klutsch, J.G., Tavener, S.J., Antolin, M.F., 2012. Demographic projection of high-elevation white pines infected with white pine blister rust: a nonlinear disease model. Ecological Applications 22, 166-183.

Fitt, B.D.L., Gregory, P.H., Todd, A.D., McCartney, H.A., Macdonald, O.C., 1987. Spore dispersal and plant disease gradients; a comparison between two empirical models. Journal of Phytopathology 118, 227-242.

Frank, K.L., Geils, B.W., Kalkstein, L.S., Thistle, Jr., H.W., 2008. Synoptic climatology of the long-distance dispersal of white pine blister rust II. Combination of surface and upper-level conditions. International Journal of Biometeorology 52, 653-666. doi:10.1007/s00484-008-0158-3.

Geils, B.W., Hummer, K.E., Hunt, R.S., 2010. White pines, Ribes, and blister rust: a review and synthesis. Forest Pathology 40, 147-185. doi:10.1111/j.1439-0329.2010.00654.x.

Giffin, W.C., 1975. Transform Techniques for Probability Modeling. Academic Press, New York.

Güssow, H.T., 1922. Interim Report of the Dominion Botanist: For the Year Ending March 31, 1922. Division of Botany, Department of Agriculture, Dominion of Canada, F.A. Acland, Ottawa. 
Kimmey, J.W., Wagener, W.W., 1961. Spread of white pine blister rust from Ribes to sugar pine in California and Oregon. Technical Bulletin 1251, U.S. Department of Agriculture, Forest Service, Washington, DC.

Kot, M., 1992. Discrete-time travelling waves: ecological examples. Journal of Mathematical Biology 30, 413-436.

Kot, M., Lewis, M.A., van den Driessche, P., 1996. Dispersal data and the spread of invading organisms. Ecology 77, 2027-2042. doi:10.2307/2265698.

Kot, M., Neubert, M.G., 2008. Saddle-point approximations, integrodifference equations, and invasions. Bulletin of Mathematical Biology 70, 1790-826. doi:10.1007/s11538-008-9325-2.

Kot, M., Schaffer, W.M., 1984. The effects of seasonality on discrete models of population growth. Theoretical Population Biology 26, 340-360. doi:10.1016/0040-5809(84)90038-8.

Lachmund, H.G., 1933. Mode of entrance and periods in the life cycle of Cronartium ribicola on Pinus monticola. Journal of Agricultural Research 47, 791805 .

Lundquist, J.E., Geils, B.W., Johnson, D.W., 1992. White pine blister rust on limber pine in South Dakota. Plant Disease 76, 538. doi:10.1094/PD-760538A.

Maloy, O.C., 1997. White pine blister rust control in North America: a case history. Annual Review of Phytopathology 35, 87-109.

McDonald, G.I., Richardson, B.A., Zambino, P.J., Klopfenstein, N.B., Kim, M.S., 2006. Pedicularis and Castilleja are natural hosts of Cronartium ribicola in North America: a first report. Forest Pathology 36, 73-82. doi:10.1111/j.1439-0329.2006.00432.x.

Medlock, J., Kot, M., 2003. Spreading disease: integro-differential equations old and new. Mathematical Biosciences 184, 201-22.

Mielke, J.L., 1943. White pine blister rust in western North America. Yale University School of Forestry Bulletin 52, 1-155.

Mollison, D., 1972. The rate of spatial propagation of simple epidemics, in: Proceedings of the Sixth Berkeley Symposium on Mathematical Statistics and Probability, Volume 3: Probability Theory, University of California Press, Berkeley, CA. pp. 579-614.

Okubo, A., Levin, S.A., 1989. A theoretical framework for data analysis of wind dispersal of seeds and pollen. Ecology 70, 329-338. doi:10.2307/1937537.

Pennington, L.H., 1925. Relation of weather conditions to the spread of white pine blister rust in the Pacific Northwest. Journal of Agricultural Research 30, 593-607. 
Posey, G.B., Ford, E.R., 1924. Survey of blister rust infection on pines at Kittery Point, Maine and the effects of Ribes eradication in controlling the disease. Journal of Agricultural Research 28, 1253-1258.

Powell, J.A., Zimmermann, N.E., 2004. Multiscale analysis of active seed dispersal contributes to resolving Reid's paradox. Ecology 85, 490-506. doi:10.1890/02-0535.

Ríos-Soto, K.R., Castillo-Chávez, C., Neubert, M.G., Titi, E.S., Yakubu, A.A., 2006. Epidemic spread in populations at demographic equilibrium, in: Gumel, A.B., Castillo-Chávez, C., Mickens, R.E., Clemence, D.P. (Eds.), Mathematical studies on human disease dynamics. American Mathematical Society, Providence, RI. volume 410 of Contemporary Mathematics, pp. 297-309. URL: http://lccn.loc.gov/2006042817.

Scheffer, R.P., 1997. The Nature of Disease in Plants. Cambridge University Press, Cambridge, UK.

Schwandt, J.W., Kearns, H.S.J., Marsden, M.A., Byler, J.W., 2013. White Pine Blister Rust Canker Expansion on Improved Western White Pine in Northern Idaho: Implications for the Management of Rust Resistant Stock. Technical Report 13-03. USDA Forest Service, Northern Region. Missoula, MT.

Spaulding, P., 1922. Investigations of the white-pine blister rust. United States Department of Agriculture Bulletin 957, 1-100.

Tomback, D.F., Achuff, P., 2010. Blister rust and western forest biodiversity: ecology, values and outlook for white pines. Forest Pathology 40, 186-225. doi:10.1111/j.1439-0329.2010.00655.x.

Tomback, D.F., Arno, S.F., Keane, R.E. (Eds.), 2001. Whitebark Pine Communities: Ecology and Restoration. Island Press, Washington, DC.

Van Arsdel, E.P., 1965. Relationships between night breezes and blister rust spread on Lake States white pines. Res. Note LS-60, USDA Forest Service, Lake States Forest Experiment Station, St. Paul, MN.

Zambino, P.J., 2010. Biology and pathology of Ribes and their implications for management of white pine blister rust. Forest Pathology 40, 264-291. doi:10.1111/j.1439-0329.2010.00658.x. 